ISSN: 2386-3919 - e-ISSN: 2386-3927

DOI: https://doi.org/10.14201/et2016342149167

\title{
EVALUACIÓN DE OBJETOS DE APRENDIZAJE EN REALIDAD AUMENTADA: ESTUDIO PILOTO EN EL GRADO DE MEDICINA
}

\section{Evaluation of objects of learning in Augmented Reality: pilot study in the degree of Medicine}

\author{
Julio Barroso OSunA* y Julio CABERO Almenara** \\ Universidad de Sevilla \\ *Correo-e: jbarroso@us.es \\ *Correo-e: cabero@us.es
}

Recibido: 2/4/2016; Aceptado: 26/9/2016; Publicado: 30/11/2016

Ref. Bibl. JULIO BARROSO OSUNA y JULIO CABERO ALMENARA. Evaluación de objetos de aprendizaje en Realidad Aumentada: estudio piloto en el Grado de Medicina. Enseñanza \& Teaching, 34, 2-2016, 149-167.

RESUMEN: El artículo gira en torno a la utilización educativa de contenidos en Realidad Aumentada en contextos de formación universitaria, tecnología que es considerada emergente y que tendrá un fuerte nivel de penetración en nuestros centros educativos y universidades a un cercano horizonte. Con el objeto de analizar las percepciones que los alumnos mostraban respecto a la calidad técnica y estética, y su facilidad de utilización, de objetos producidos para los estudiantes del Grado de Medicina que cursaban la asignatura de "Anatomía Humana I», impartida en el primer curso de dicho Grado, se realizó un estudio piloto donde los estudiantes interaccionaron con los mismos y finalmente tenían que evaluarlos, para ello se creó y fiabilizó un instrumento específico. Los resultados encontrados muestran diferentes aspectos que van desde las percepciones significativas que los objetos producidos han despertado en los estudiantes, su facilidad de uso y su posibilidad de integrarse en la enseñanza. Los resultados encontrados coinciden en los alcanzados en otros trabajos. 
JULIO BARROSO OSUNA Y JULIO CABERO ALMENARA

EVALUACIÓN DE OBJETOS DE APRENDIZAJE EN REALIDAD AUMENTADA ESTUDIO PILOTO EN EL GRADO DE MEDICINA

Palabras clave: realidad aumentada; diseño, producción y evaluación de medios y materiales; experiencia en el aula; integración curricular de las TIC; tecnologías emergentes.

SUMMARY: The article turns around the educational utilization of contents in Augmented Reality (AR) in contexts of universitary education, technology which is considered emergent and that will have a strong level of penetration in our educational centers and universities to a nearby horizon. In order to analyze the perceptions that the pupils were showing with regard to the technical and aesthetic quality, and the facility of utilization, of objects produced for the students of the degree of Medicine who were dealing the subject of "Human Anatomy I", given in the first course of the above mentioned Degree, a pilot study was realized where the students intergesticulated with the same ones and finally they had to evaluate them, for it, it was created and reliabited a specific instrument. The opposing results show different aspects that go from: the significant perceptions that the produced objects have woken up in the students, its facility of use and its possibility of joining the education. The opposing results coincide with the reached one in other works.

Key words: augmented reality; design, production and evaluation of means and materials; experience in the classroom; integration curricular of the TIC; emergent technologies.

\section{LA REALIDAD AUMENTADA: TECNOLOGÍA EMERGENTE PARA LA FORMACIÓN}

Nos encontramos ante nuevos escenarios de formación apoyados en una fuerte presencia de las Tecnologías de la Información y Comunicación (TIC), que, como nos indica Cabero (2016), van a reclamar nuevos aspectos que han de ser tenidos en cuenta a lo largo del proceso educativo: la necesidad del aumento de interacción profesor-alumno, la incorporación de nuevas metodologías colaborativas, el papel más activo de los estudiantes, las percepciones de los estudiantes como proconsumidores y la presencia de unas "galaxias" de tecnologías que crean nuevas escenografías para que el docente genere ambientes enriquecidos tecnológicamente para el aprendizaje de los estudiantes.

A la hora de determinar cuáles son las tendencias en tecnologías aplicadas a la educación que se consideran emergentes en la actualidad, podemos prestar atención a las diferentes publicaciones especializadas (libros, revistas, blogs, boletines de noticias, etc.) o centrarnos en informes como los siguientes: el informe de innovación pedagógica de la Open University de Reino Unido (OU) (http://www. open.ac.uk/blogs/innovating/), el informe 2014 sobre educación y tecnología de la fundación Gold Mercury International, los «informe Horizon», los Informes, los "Reportes EduTrends» del Observatorio de Innovación Educativa Tecnológico de Monterrey (http://observatorio.itesm.mx/edutrendsradar2015/) o los hiperciclos 
de aceptación de cualquier tecnología realizados por la compañía Gartner (http:// www.gartner.com/technology/home.jsp).

Nosotros nos centraremos en el «informe Horizon", proyecto creado en 2002 por el NMC (New Media Consortium, http://nmc.org) con el que se pretende informar de las tecnologías a las que hay que prestar atención a corto ( 1 año o menos), medio (2-3 años) y largo plazo (4-5 años) para ser incorporadas al sector educativo. En estos documentos podemos observar como una de las tecnologías que en los últimos tiempos se está presentando como emergente y con verdaderas posibilidades de impactar en el terreno educativo es la denominada "Realidad Aumentada" ("Augmented Reality») (RA), tecnología que, de acuerdo con diferentes informes Horizon (Johnson, Adams Becker, Cummins, Estrada, Freeman y Hall, 2016), tendrá una fuerte penetración en las instancias educativas en un horizonte de 3 a 5 años.

Esta significación podemos también intuirla mediante el empleo de la herramienta "Ngram Vieewer" de Google, que nos ofrece la evolución de ciertos términos en los documentos que la citada institución ha digitalizado. En la Figura 1, el lector puede observar la evolución en los últimos años de las publicaciones que incorporaron los términos "Realidad Aumentada» y "Augmented Reality».

FIGURA 1

Curva ofrecida por Google «Ngram Vieewer» para los términos "Realidad Aumentada» y "Augmented Reality"

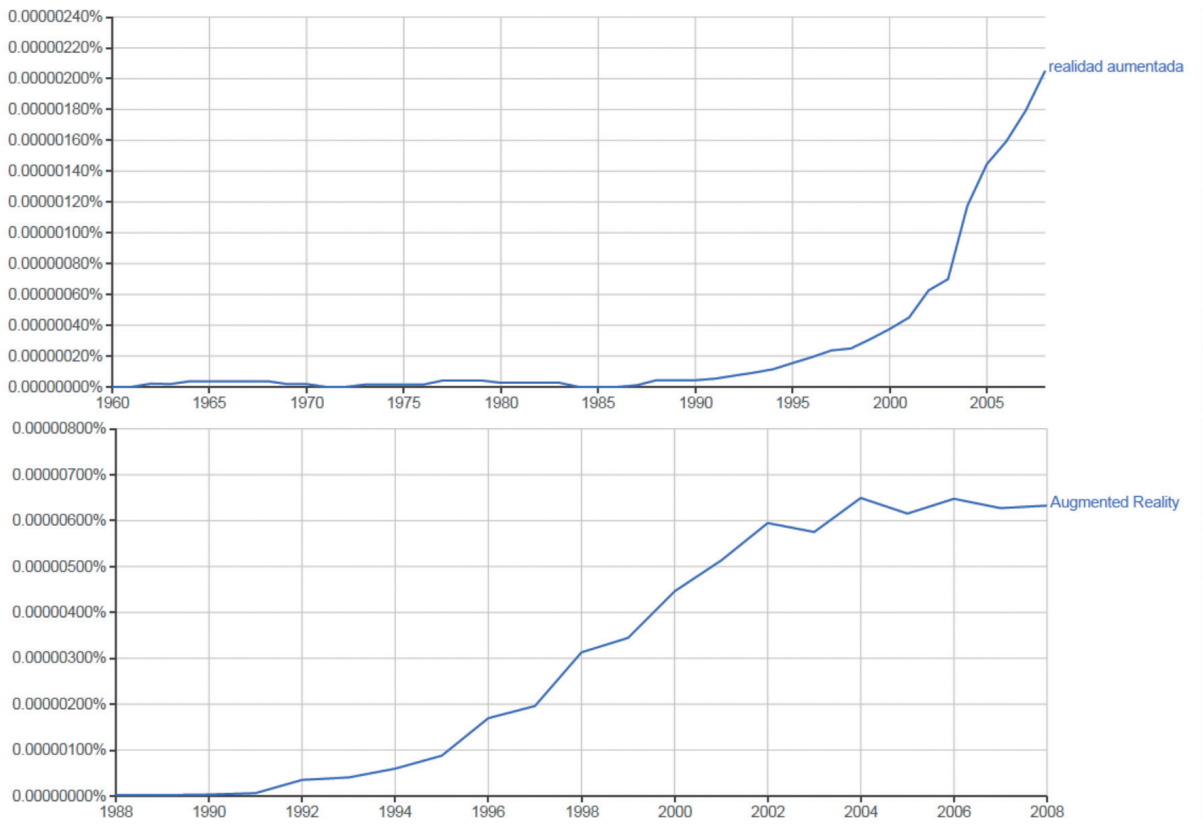


Ahora bien, ¿qué podemos entender por RA?, y al respecto y de acuerdo con las propuestas de diferentes autores (Fundación Telefónica, 2011; Fombona, Pascual y Madeira, 2012; Muñoz, 2013; Cabero y Barroso, 2015, 2016; Prendes, 2015; Cabero y García, 2016), podemos indicar que es la combinación de información digital e información física en tiempo real a través de diferentes dispositivos tecnológicos; es decir, consiste en utilizar un conjunto de dispositivos tecnológicos que añaden información virtual a la información física, para crear con ello una nueva realidad, pero donde tanto la información real como la virtual desempeñan un papel significativo.

Otra forma de comprender lo que es la RA es diferenciarla de la "Realidad Virtual" (RV), en la cual no se combina la realidad con elementos informativos ubicados en los dispositivos tecnológicos para alcanzar con ello una nueva realidad, sino que en este caso el sujeto se ve incorporado en un contexto inmersivo tecnológico y no se ve mezclado con la realidad (Dias, 2016; Johnson et al., 2016).

Recientemente, en una obra coordinada por Cabero y García (2016), se han llegado a señalar algunas de las propiedades más significativas de la RA y que las concretan en las siguientes: es una realidad mixta, integrada coherentemente en tiempo real, que posee una diversidad de capas de información digital, que es interactiva y que mediante su utilización enriquecemos o alteramos la información.

Esta tecnología está adquiriendo una progresiva penetración en el campo educativo en los últimos tiempos, desarrollándose experiencias en los diferentes niveles educativos, que van desde el de primaria (Bongiovani, 2013; Prendes, 2015), secundaria-bachillerato-formación profesional (Liu, 2009; Avendaño, Chao, Mercado y Bazán, 2012; De Pedro Carracedo y Méndez, 2012; Bressler y Bodzin, 2013; De la Torre, Martín-Dorta, Saorín, Carbonel y González, 2013) y universitario (Rodríguez, 2013).

Al mismo tiempo sus aplicaciones se están ejecutando en diferentes áreas curriculares: ingeniería (De la Torre et al., 2013), arquitectura (De la Torre et al., 2013), urbanismo (Carozza, Tingdahl, Gool, Bosché y Gool, 2014), matemáticas-geometría (Avendaño et al., 2012; De Pedro Carracedo y Méndez, 2012; Coimbra, Cardoso y Mateus, 2015), arte e historia (Ruiz, 2011), aprendizaje de idiomas (Liu, 2009; Santos et al., 2015), tecnología (Rodríguez, 2013), diseño (Ko, Chang, Chen y Hua, 2011), química (Pasaréti, Hajdú, Matuszka, Jambori, Molnar y Turcsányi-Szabó, 2011), ciencias naturales (Fracchia, Alonso y Martins, 2015), biología (Solano, Casas y Guevara, 2015), geografía (Tsai, Liu y Yau, 2013).

En el caso que nos ocupa, el de la medicina, se han venido realizando diferentes propuestas de utilización en esta disciplina para aumentar la comprensión y motivación de los estudiantes, hacia los diferentes contenidos presentados (Mott et al., 2008; Fundación Telefónica, 2011; Yeo, Ungi, U-Thainual, Lasso, McGraw y Fichtinger, 2011; Wu, Wen-Yu, Chang y Liang, 2013; Bower et al., 2014).

Al mismo tiempo, debemos reconocer que su extensión ha sido posible gracias a la significación que los dispositivos móviles están adquiriendo en nuestra sociedad, que favorecen el desarrollo del aprendizaje ubicuo en los contextos de formación (Quicios, Ortega y Trillo, 2015; Sevillano y Vázquez, 2015; Vázquez y Sevillano, 2015).

En cuanto a las investigaciones que se han llevado a cabo, aunque aún son escasas, han puesto de manifiesto que los alumnos muestran actitudes favorables 
hacia ella y que su utilización aumenta la motivación hacia el aprendizaje (Bressler y Bodzin, 2013; Kamarainen et al., 2013; Di Serio, Ibáñez y Delgado, 2013; Cózar, De Moya, Hernádez y Hernández, 2015), que favorecen la creación de un contexto constructivista de formación (Chen y Tsai, 2012; Wojciechowski y Cellary, 2013), que propicia un entorno activo de enseñanza (Fombona et al., 2012), que despierta un alto grado de satisfacción cuando los alumnos participan en estas experiencias (Han, Jo, Hyun y So, 2015), y que su utilización mejora los resultados de aprendizajes (Bongiovani, 2013; Chang, Wu, \& Hsu, 2013; Kamarainen et al., 2013).

En este artículo incluimos los datos obtenidos a través de un estudio piloto de un proyecto de $\mathrm{I}+\mathrm{D}+\mathrm{i}$ del Ministerio de Economía y Competitividad del Gobierno de España denominado: "Realidad aumentada para aumentar la formación. Diseño, producción y evaluación de programas de realidad aumentada para la formación universitaria” (EDU-5746-P - Proyecto Rafodiun). Evolución del proyecto que el lector interesado por la temática de la RA puede seguir, por una parte, en la web que se ha creado del mismo (http://intra.sav.us.es/proyectorafodiun/) o en la comunidad virtual de análisis de las posibilidades educativas de la tecnología de la que hablamos (https://plus.google.com/u/0/communities/102143147822806126247).

\section{LA PRODUCCIÓN DE OBJETOS DE APRENDIZAJE EN RA: DISEÑO Y PRODUCCIÓN}

Para la producción de los objetos en RA se pueden utilizar diferentes tipos de programas: Layar, Metaio, Junaio, Augment, Aurasma, Unity...; en nuestro caso para la producción de los objetos que utilizamos en nuestra investigación se utilizaron como herramientas fundamentales "Metaio Creator" y "Metaio SDK". La programación necesaria para obtener las apps que permitirían utilizar los objetos se basó en AREL (Augmented Reality Experience Language), que funciona gracias a una combinación de código javascript y una definición de los objetos basados en un documento XML (Extended Markup Language).

FIGURA 2

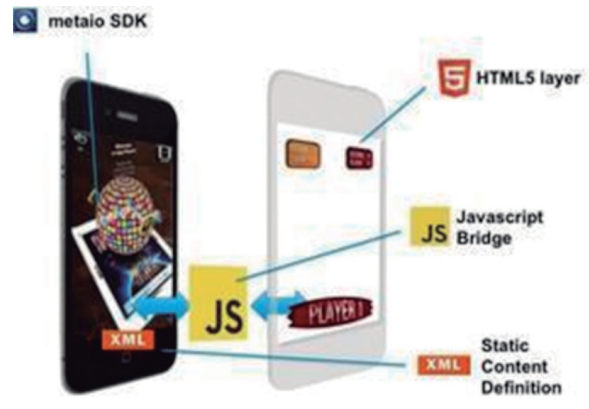

Fuente: http://dev.metaio.com/arel/overview/ 
Los cuatro objetos que se han utilizado en el presente estudio: Shoulder, Coxofemoral, Cervical y Ankle, permiten que los alumnos puedan observar las partes óseas de estos componentes de la anatomía humana en 3D; ampliarlos; desplazarlos por la pantalla; observarlos desde diferentes puntos de vista, pues los objetos se presentan en constante rotación, e ir a vídeos explicativos ubicados en Youtube. En todo momento es el alumno el que controla las acciones a realizar con el objeto. Indicar que tales objetos estaban incorporados en unos apuntes ofrecidos a los estudiantes y, por tanto, los objetos en RA cumplían la función de ampliar y enriquecer la información que se presentaba en los apuntes, sin la necesidad de su abandono por parte de los estudiantes.

Los cuatro objetos fueron producidos desde el punto de vista de contenido y diseño didáctico por los profesores de la Universidad de Sevilla: Manuel Eugenio Dorado Ocaña, Emilio Gómez González y Javier Márquez Rivas; y por lo que se refiere a su diseño técnico por Fernando García Jiménez, Inés Casado, Óscar Gallego, Ángel Martín y Silvia Piña Fiol, del SAV de la Universidad de Sevilla.

Señalar que en el momento de realizar la experiencia las app solo estaban disponibles para los dispositivos Android, pero que en la actualidad ya están disponibles para los dispositivos IOS.

Los objetos producidos para nuestra experiencia presentan una serie de características: ofrecen animaciones en 3D de diferentes partes del esqueleto humano, permiten la parada y seguimiento del objeto por parte del estudiante, posibilitan la ampliación del tamaño del componente que es presentado para que puedan ser observados los detalles por parte de los estudiantes y transfieren al estudiante a un vídeo ampliador de la información que ha sido ubicado en Youtube para facilitar la rapidez del funcionamiento del objeto y evitar la demora del tiempo de descarga.

En la Figura 3, se presentan una serie de imágenes de los objetos producidos en RA para la investigación.

\section{FIGURA 3}

Objetos producidos en RA para la investigación
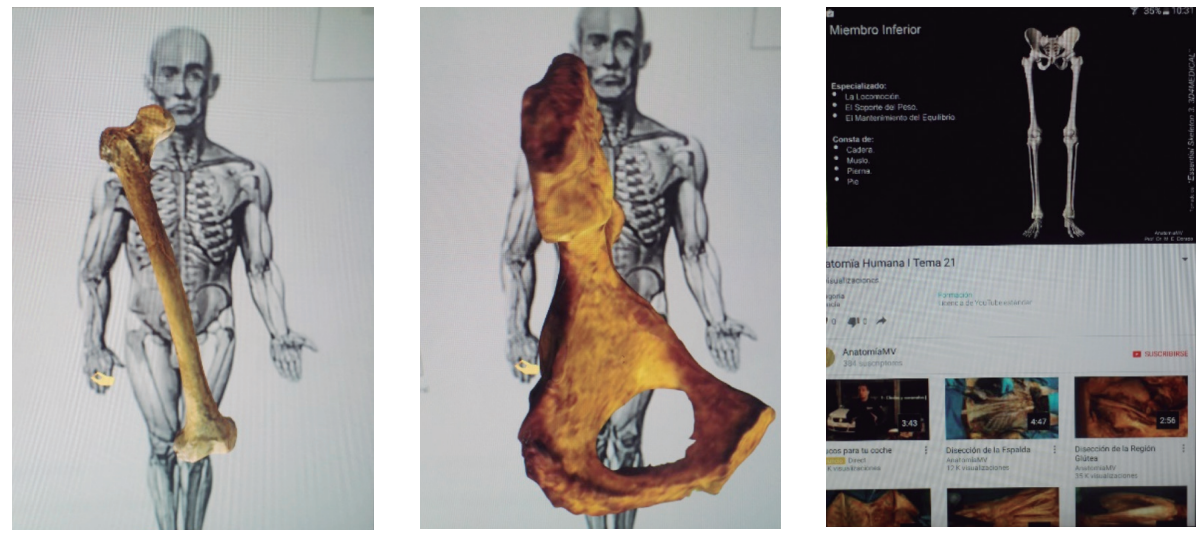


\section{EL ESTUDIO REALIZADO}

El proyecto de investigación realizado se enmarca dentro de un proyecto de investigación financiado por el Ministerio de Economía y Competividad de España denominado RAFODIUN (EDU2014-57446-P); en el cual uno de los objetivos perseguidos era: "Diseñar y producir distintos contenidos en formato RA para ser aplicados en contextos de formación universitaria en distintas áreas curriculares, y evaluar sus posibilidades de cara al rendimiento de los alumnos». Por ello, una vez producidos y con el objeto de recoger la opinión y valoración de los alumnos a los cuales estaban dirigidos los objetos de aprendizaje en RA que elaboramos, llevamos a cabo un estudio piloto con alumnos del Grado de Medicina que cursaban la asignatura de "Anatomía Humana I» de primer curso y que es impartida por el Departamento de "Anatomía y Embriología Humana». La investigación se llevó a cabo en los grupos que cursaban la asignatura en las cuales impartían docencia los profesores que habían participado en la producción de los materiales de enseñanza en RA, y que conformaban más del cincuenta por ciento de los grupos que recibían esta asignatura. El número de participantes, una vez eliminados aquellos que no cumplimentaron correctamente los instrumentos, fue de 50, de los cuales 21 (42\%) era hombres y 29 (58\%) mujeres.

Por tanto, podemos señalar que la muestra utilizada en nuestra investigación ha sido no probabilística y del tipo de conveniencia o causal (Alamino, 2006; Sabariego, 2012) que se basa en la facilidad de acceso por parte del investigador a los individuos que participaron en el estudio.

El instrumento de recogida de información fue un cuestionario con construcción tipo Likert, conformado por 13 ítems con seis opciones de respuestas $(\mathrm{MP}=$ Muy positivo/Muy de acuerdo; $\mathrm{P}=$ Positivo/De acuerdo; $\mathrm{R}+=$ Regular positivo/ Moderadamente de acuerdo; R-= Regular negativo/Moderadamente en desacuerdo; $\mathrm{N}=$ Negativo/En desacuerdo; y MN= Muy negativo/Muy en desacuerdo), que pretendían recoger información sobre tres dimensiones, dos referidas directamente al objeto producido: aspectos técnicos y estéticos ( 4 ítems), y facilidad de utilización (7 ítems); y una destinada a la valoración de la guía que para la comprensión del funcionamiento del objeto por parte de los usuarios habíamos elaborado. En la Figura 4, se presenta un ejemplo de cómo era presentado el material a los estudiantes, en concreto el del hombro (shoulder); en la página web del proyecto (http://intra.sav.us.es/proyectorafodiun/index.php), pueden observarse los diferentes objetos que se produjeron para este estudio. 

ESTUDIO PILOTO EN EL GRADO DE MEDICINA

FIGURA 4

Guía objeto Shoulder

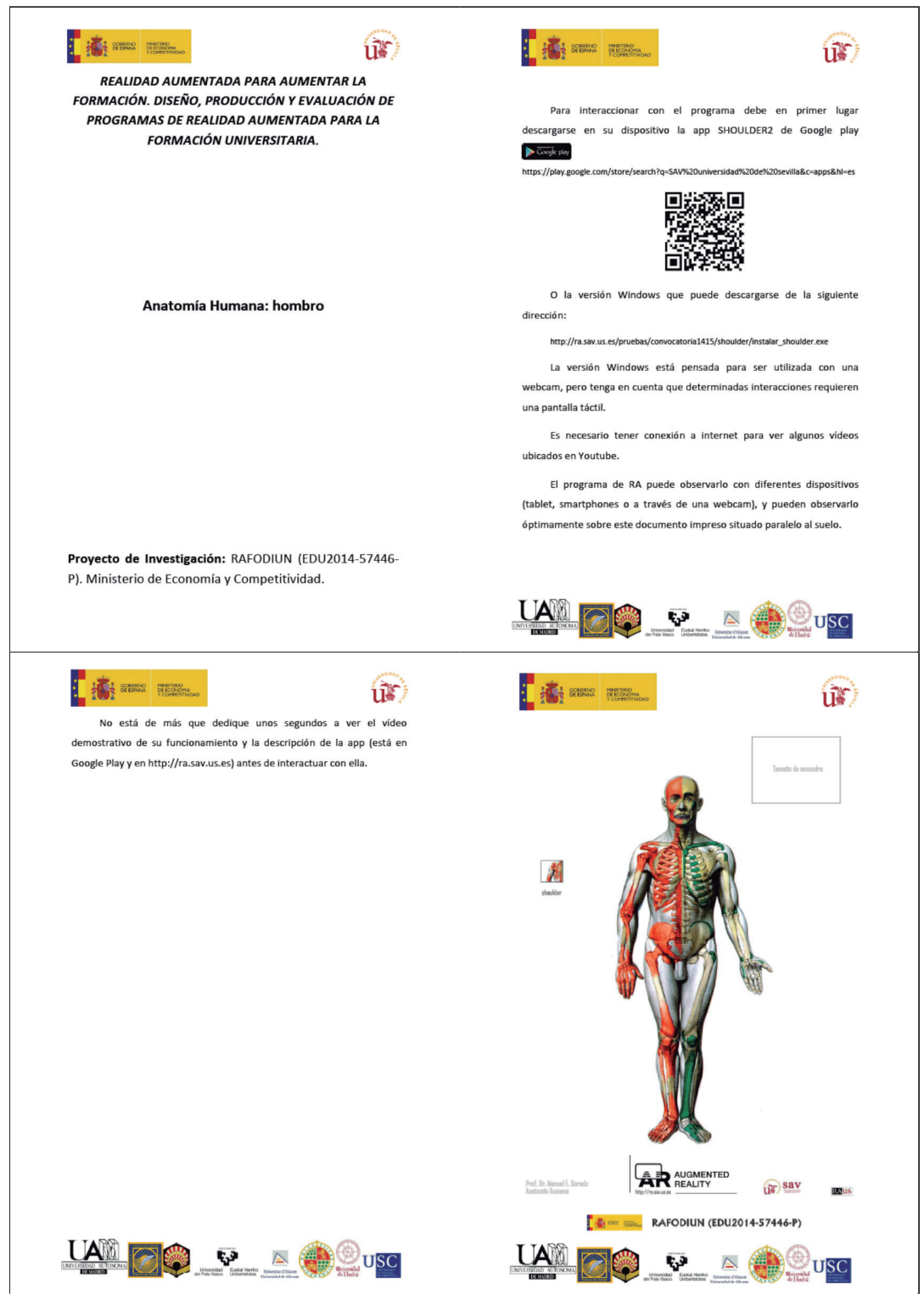


La guía pretendía ofrecerles a los alumnos información respecto a diferentes aspectos: lugar desde el cual podrían bajarse la app para la utilización del programa, dispositivos que podrían utilizar para la interacción con el objeto y recomendaciones para la observación de vídeos demostrativos del funcionamiento que se realizaron para facilitar la comprensión de su utilización y manejo.

La evaluación de los materiales se llevó a cabo en una sesión donde se realizaron las siguientes acciones:

a) Explicación de en qué consistía la RA.

b) Demostración de los diferentes objetos producidos.

c) Presentación del lugar del cual podrían bajarse las distintas guías de los cuatro objetos que se les iban a presentar y que posteriormente deberían evaluar (shoulder, coxofemoral, cervical y ankle) (http://intra.sav.us.es/ proyectorafodiun/index.php/objetos-en-ra).

d) Trabajo individual de los alumnos con sus dispositivos móviles para interaccionar con los diferentes objetos presentados.

e) Y cumplimentación de los cuestionarios de evaluación de los objetos de aprendizajes presentados por parte de los estudiantes.

Señalar que la sesión duró dos horas.

El cuestionario se administró vía Internet y se elaboró a partir de los producidos en otras investigaciones para la evaluación de objetos de aprendizaje de materiales multimedia, de materiales para la formación bajo la modalidad de e-learning y de objetos producidos para un entorno virtual de formación bajo la estructura de «Entorno Personal de Aprendizaje» (Cabero y Llorente, 2009; Llorente y Cabero, 2013; Cabero y Marín, 2013; Marín, Cabero y Barroso, 2014).

Con objeto de analizar su índice de fiabilidad, aplicamos el estadístico alfa de Cronbach que, de acuerdo con O'Dwyer y Bernauer (2014), es el más apropiado para este tipo de instrumentos, alcanzando un valor del 0,941, que, de acuerdo con la propuesta de Mateo (2004), se puede considerar de alta o muy alta. Con objeto de conocer si la eliminación de algún ítem aumentaría el nivel de fiabilidad del instrumento, aplicamos la correlación ítem-total, obteniéndose los valores que presentamos en la Tabla 1. 
JULIO BARROSO OSUNA Y JULIO CABERO ALMENARA EVALUACIÓN DE OBJETOS DE APRENDIZAJE EN REALIDAD AUMENTADA: ESTUDIO PILOTO EN EL GRADO DE MEDICINA

TABLA 1

Correlación ítem-total

\begin{tabular}{|c|c|c|c|c|}
\hline & $\begin{array}{c}\text { MEDIA DE ESCALA SI } \\
\text { EL ELEMENTO SE HA } \\
\text { SUPRIMIDO }\end{array}$ & $\begin{array}{c}\text { VARIANZA DE ESCALA } \\
\text { SI EL ELEMENTO SE HA } \\
\text { SUPRIMIDO }\end{array}$ & $\begin{array}{c}\text { CORRELACIÓN TOTAL } \\
\text { DE ELEMENTOS } \\
\text { CORREGIDA }\end{array}$ & $\begin{array}{c}\text { ALFA } \\
\text { DE CRONBACH SI } \\
\text { EL ELEMENTO SE HA } \\
\text { SUPRIMIDO }\end{array}$ \\
\hline 1.1 & 203,26 & 420,972 & 0,726 & 0,938 \\
\hline 1.2 & 203,50 & 423,520 & 0,556 & 0,939 \\
\hline 1.3 & 203,50 & 429,357 & 0,350 & 0,941 \\
\hline 1.4 & 203,28 & 410,859 & 0,864 & 0,937 \\
\hline 2.1 & 203,38 & 430,812 & 0,386 & 0,941 \\
\hline 2.2 & 203,12 & 424,924 & 0,625 & 0,939 \\
\hline 2.3 & 203,20 & 416,776 & 0,889 & 0,937 \\
\hline 2.4 & 203,38 & 417,628 & 0,490 & 0,940 \\
\hline 2.5 & 203,04 & 425,427 & 0,450 & 0,940 \\
\hline 2.6 & 203,20 & 424,857 & 0,581 & 0,939 \\
\hline 3.1 & 203,18 & 427,498 & 0,445 & 0,940 \\
\hline 3.2 & 203,24 & 423,411 & 0,648 & 0,939 \\
\hline
\end{tabular}

Como podemos observar en la tabla anterior, la eliminación de ningún ítem llevaría a ampliar ostensiblemente el valor alfa global anteriormente indicado; por ello se tomó la decisión de no eliminar ninguno de ellos y mantener el instrumento en la forma en la cual fue administrado a los alumnos.

\section{RESULTADOS ENCONTRADOS}

Para facilitar la comprensión de los resultados alcanzados, ofreceremos en primer lugar las valoraciones medias globales obtenidas con el instrumento para los cuatro objetos con que interaccionaron los estudiantes, y la puntuación media global alcanzada para cada uno de los objetos; para ello asignamos la puntuación de 6 a la opción de respuesta de MP= Muy positivo/Muy de acuerdo y 1 a MN= Muy negativo/Muy en desacuerdo. En la Tabla 2, presentamos los valores medios y las desviaciones típicas alcanzadas. 
JULIO BARROSO OSUNA Y JULIO CABERO ALMENARA

EVALUACIÓN DE OBJETOS DE APRENDIZAJE EN REALIDAD AUMENTADA: ESTUDIO PILOTO EN EL GRADO DE MEDICINA

TABLA 2

Puntuaciones medias y desviaciones típicas totales alcanzadas

\begin{tabular}{|l|c|c|}
\hline \multicolumn{1}{|c|}{ OBJETO } & MEDIA & DT \\
\hline Todos los objetos & 4,33 & 0,44 \\
\hline Objeto Shoulder & 4,43 & 0,58 \\
\hline Objeto Coxofemoral & 4,36 & 0,49 \\
\hline Objeto Cervical & 4,29 & 0,44 \\
\hline Objeto Ankle & 4,23 & 0,40 \\
\hline
\end{tabular}

Como podemos observar en la tabla anterior las valoraciones, tanto globales como para cada uno de los objetos analizados, se sitúan con valores relativamente superiores al nivel "Regular positivo/Moderadamente de acuerdo", con desviaciones típicas no muy elevadas; lo que denota cierto grado de unificación de las valoraciones asignadas por los alumnos a los objetos producidos y cierto acuerdo en el nivel de respuesta seleccionado por los estudiantes.

Por lo que se refiere a las valoraciones medias obtenidas en cada una de las dimensiones que conformaban el instrumento de evaluación utilizado, las presentamos en la Tabla 3.

TABLA 3

Puntuaciones medias y desviaciones típicas totales alcanzadas para cada una de las dimensiones

\begin{tabular}{|l|c|c|c|c|c|c|}
\hline \multicolumn{1}{|c|}{ DIMENSIÓN } & \multicolumn{2}{c|}{$\begin{array}{c}\text { ASPECTOS TÉCNICOS } \\
\text { Y ESTÉTICOS }\end{array}$} & \multicolumn{2}{c|}{$\begin{array}{c}\text { FACILIDAD } \\
\text { DE UTILIZACIÓN }\end{array}$} & \multicolumn{2}{c|}{$\begin{array}{c}\text { GUÍA/TUTORIAL } \\
\text { DEL PROGRAMA }\end{array}$} \\
\hline Objeto & Media & DT & Media & DT & Media & DT \\
\hline Todos los objetos & 4,36 & 0,60 & 4,30 & 0,53 & 4,34 & 0,71 \\
\hline Objeto Shoulder & 4,32 & 0,70 & 4,48 & 0,61 & 4,49 & 0,80 \\
\hline $\begin{array}{l}\text { Objeto } \\
\text { Coxofemoral }\end{array}$ & 4,37 & 0,62 & 4,36 & 0,56 & 4,33 & 0,69 \\
\hline Objeto Cervical & 4,46 & 0,69 & 4,18 & 0,52 & 4,26 & 0,39 \\
\hline Objeto Ankle & 4,30 & 0,49 & 4,18 & 0,47 & 4,28 & 0,62 \\
\hline
\end{tabular}

De nuevo nos encontramos con que las puntuaciones medias alcanzadas se situaron ligeramente superior a la opción de respuesta: "Regular positivo/Moderadamente de acuerdo"; y ello se ha dado tanto en las tres dimensiones que analizaban nuestro instrumento (aspectos técnicos y estéticos, facilidad de utilización y guía/tutorial del programa) como en los cuatro objetos producidos.

Una vez presentados los valores globales alcanzados para todos y para cada una de las dimensiones, pasaremos a ofrecer las puntuaciones medias obtenidas en los diferentes ítems. En la Tabla 4, se presentan las puntuaciones 
JULIO BARROSO OSUNA Y JULIO CABERO ALMENARA

EVALUACIÓN DE OBJETOS DE APRENDIZAJE EN REALIDAD AUMENTADA:

ESTUDIO PILOTO EN EL GRADO DE MEDICINA

medias globales alcanzadas en los diferentes ítems y sus desviaciones típicas correspondientes.

TABLA 4

Puntuaciones medias y desviaciones típicas totales alcanzadas para cada una de las dimensiones

\begin{tabular}{|c|c|c|}
\hline \multicolumn{3}{|l|}{ 1. ASPECTOS TÉCNICOS Y ESTÉTICOS } \\
\hline & Media & DT \\
\hline 1.1. El funcionamiento del recurso en RA que te hemos presentado es: & 4,43 & 0,88 \\
\hline 1.2. En general, la estética del recurso producido en RA lo consideras: & 4,20 & 0,81 \\
\hline $\begin{array}{l}\text { 1.3. En general, el funcionamiento técnico del recurso producido en RA } \\
\text { lo calificarías de: }\end{array}$ & 4,69 & 0,93 \\
\hline $\begin{array}{l}\text { 1.4. En general, cómo valorarías la presentación de la información en la } \\
\text { pantalla: }\end{array}$ & 4,36 & 0,87 \\
\hline \multicolumn{3}{|l|}{ 2. FACILIDAD DE UTILIZACIÓN } \\
\hline $\begin{array}{l}\text { 2.1. Cómo calificarías la facilidad de utilización y manejo del recurso en } \\
\text { RA que te hemos presentado: }\end{array}$ & 4,39 & 0,80 \\
\hline $\begin{array}{l}\text { 2.2. Cómo calificarías la facilidad de comprensión del funcionamiento } \\
\text { técnico del recurso en RA que te hemos presentado: }\end{array}$ & 4,36 & 0,83 \\
\hline $\begin{array}{l}\text { 2.3. Desde tu punto de vista, cómo valorarías el diseño general del } \\
\text { recurso en RA que hemos elaborado: }\end{array}$ & 4,27 & 0,90 \\
\hline $\begin{array}{l}\text { 2.4. Desde tu punto de vista, cómo valorarías la accesibilidad/usabilidad } \\
\text { del recurso en RA que te hemos presentado: }\end{array}$ & 4,12 & 0,96 \\
\hline $\begin{array}{l}\text { 2.5. Desde tu punto de vista, cómo valorarías la flexibilidad de utilización } \\
\text { del material en RA que te hemos presentado: }\end{array}$ & 4,38 & 0,87 \\
\hline 2.7. El utilizar el recurso en RA producido te fue divertido: & 4,30 & 0,74 \\
\hline \multicolumn{3}{|l|}{ 3. GUÍA/TUTORIAL DEL PROGRAMA } \\
\hline $\begin{array}{l}\text { 3.1. En general, cómo calificarías de eficaz y comprensible la información } \\
\text { ofrecida para manejar el recurso en RA que te hemos presentado: }\end{array}$ & 4,38 & 0,76 \\
\hline $\begin{array}{l}\text { 3.2. La información ofrecida para manejar el recurso en RA te fue simple } \\
\text { y comprensible: }\end{array}$ & 4,31 & 0,74 \\
\hline
\end{tabular}

Como se puede observar en la Tabla 4, las puntuaciones obtenidas en todos los ítems se sitúan por encima de la puntuación media que denotaba un estado de opinión de "Regular positivo/Moderadamente de acuerdo". Siendo los 3 ítems con mayor puntuación media los siguientes: «En general, el funcionamiento técnico del recurso producido en RA lo calificarías de» $(4,69)$, "Cómo calificarías la facilidad de utilización y manejo del recurso en RA que te hemos presentado» $(4,43) \mathrm{y}$ "El funcionamiento del recurso en RA que te hemos presentado es» (4,39); y los tres de menor puntuación los que presentamos a continuación: "Desde tu punto de vista, cómo valorarías la accesibilidad/usabilidad del recurso en RA que te hemos presentado» $(4,12)$, «En general, la estética del recurso producido en RA lo consideras» $(4,20)$ y 
"Desde tu punto de vista, cómo valorarías el diseño general del recurso en RA que hemos elaborado" $(4,27)$.

Ordenados de mayor a menor los ítems por las puntuaciones medias obtenidas la distribución que obtenemos de los ítems los presentamos en la Tabla 5.

TABLA 5

Distribución de los ítems de acuerdo a los valores medios alcanzados

\begin{tabular}{|l|c|}
\hline \multicolumn{1}{|c|}{ ÍTEMS } & MEDIA \\
\hline $\begin{array}{l}\text { 2.4. Desde tu punto de vista, cómo valorarías la accesibilidad/usabilidad del } \\
\text { recurso en RA que te hemos presentado: }\end{array}$ & 4,12 \\
\hline $\begin{array}{l}\text { 1.2. En general, la estética del recurso producido en RA lo consideras: } \\
\text { Ra que hemos elaborado: }\end{array}$ & 4,20 \\
\hline 2.7. El utilizar el recurso en RA producido te fue divertido: & 4,27 \\
\hline $\begin{array}{l}\text { 3.2. La información ofrecida para manejar el recurso en RA te fue simple y } \\
\text { comprensible: }\end{array}$ & 4,30 \\
\hline $\begin{array}{l}\text { 1.4. En general, cómo valorarías la presentación de la información en la } \\
\text { pantalla: }\end{array}$ & 4,31 \\
\hline $\begin{array}{l}\text { 2.2. Cómo calificarías la facilidad de comprensión del funcionamiento técnico } \\
\text { del recurso en RA que te hemos presentado: }\end{array}$ & 4,36 \\
\hline $\begin{array}{l}\text { 2.5. Desde tu punto de vista, cómo valorarías la flexibilidad de utilización del } \\
\text { material en RA que te hemos presentado: }\end{array}$ & 4,38 \\
\hline $\begin{array}{l}\text { 3.1. En general, cómo calificarías de eficaz y comprensible la información } \\
\text { ofrecida para manejar el recurso en RA que te hemos presentado: }\end{array}$ & 4,38 \\
\hline $\begin{array}{l}\text { 2.1. Cómo calificarías la facilidad de utilización y manejo del recurso en RA que } \\
\text { te hemos presentado: }\end{array}$ & 4,39 \\
\hline 1.1. El funcionamiento del recurso en RA que te hemos presentado es: & 4,43 \\
\hline $\begin{array}{l}\text { 1.3. En general, el funcionamiento técnico del recurso producido en RA lo } \\
\text { calificarías de: }\end{array}$ & 4,69 \\
\hline
\end{tabular}

Como podemos observar la ordenación de los ítems no nos indica que los ítems de dimensiones específicas hayan sido valorados de forma más significativa que otros, sino que se distribuyen de manera que podríamos decir no uniforme; y por tanto nos indicaría la no gran diferencia entre los ítems de las diferentes dimensiones.

En el caso de los valores alcanzados en cada uno de los objetos de forma individual las puntuaciones medias las presentamos en la Tabla 6. 

ESTUDIO PILOTO EN EL GRADO DE MEDICINA

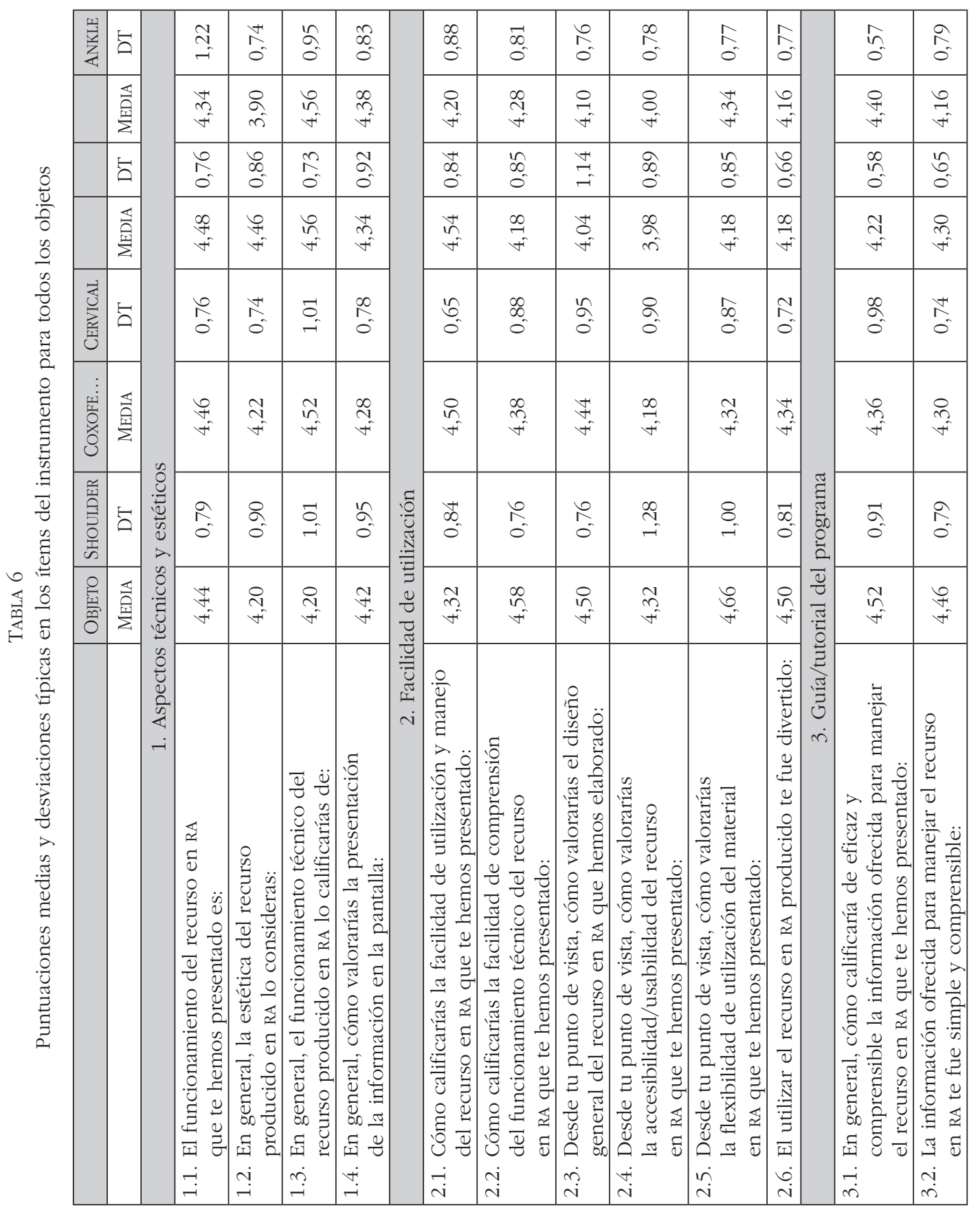


Aunque las valoraciones medias son relativamente similares para los diferentes objetos producidos, nos hemos encontrado dos casos donde los ítems fueron valorados con puntuaciones inferiores a "4», y ello ha sido con el objeto "Ankle» $(3,90)$ en el ítem «En general, la estética del recurso producido en RA lo consideras» $\mathrm{y}$ "Cervical» $(3,98)$ con el ítem «Desde tu punto de vista, cómo valorarías la accesibilidad/usabilidad del recurso en RA que te hemos presentado”. Sí nos gustaría señalar que las desviaciones típicas alcanzadas en algunos ítems son bastante elevadas y por tanto denotarían una fuerte variabilidad en las contestaciones ofrecidas por los alumnos.

\section{CONCLUSIONES, DISCUSIÓN Y LIMITACIONES DEL ESTUDIO}

El trabajo presentado permite alcanzar una serie de conclusiones generales, la primera de ellas es que los objetos de RA que se han producido han despertado verdadero interés para los estudiantes del Grado de Medicina que han participado en el estudio. Y ello tanto desde el punto de vista técnico y estético, como de su facilidad de utilización. Creemos que a esta facilidad de utilización ha ayudado la guía elaborada para que sirva de asesoramiento de su funcionamiento a los estudiantes. Lo cual nos sugiere que la incorporación de estos objetos en acciones de formación no debe limitarse a la simple indicación de las app que los estudiantes pueden «bajarse» a sus dispositivos móviles (Quicios et al., 2015; Sevillano y Vázquez, 2015; Vázquez y Sevillano, 2015).

Lo comentado nos lleva a señalar que la tecnología de la RA es fácil de incorporar a la enseñanza. Desde nuestro estudio se pueden apuntar dos aspectos: no se requirió tecnología adicional de incorporación puesto que se trabajó con los dispositivos propios de los estudiantes y no se encontraron dificultades de interacción y comprensión de su manejo por los estudiantes. Aunque también debemos reconocer que como toda tecnología novedosa en los primeros momentos crea inseguridad.

Nuestra investigación apuntala la idea expuesta por Wojciechowski y Cellary (2013), cuando sugieren que mediante estos objetos los alumnos son capaces de interactuar con los objetos virtuales en una forma directa y natural mediante la manipulación de objetos reales y sin la necesidad de dispositivos sofisticados y costosos. Y nosotros añadiríamos, además, de manera fácil y accesible, de acuerdo con las puntuaciones que nos han ofrecido los estudiantes de los objetos que han utilizado.

Indirectamente nuestros hallazgos se encuentran en consonancia con los apuntados por diferentes autores (Chin-Ming y Yen Nung, 2011; De la Torre et al., 2013; Kamarainen et al., 2013; Ko et al., 2011; Neven, Hala y Mohamed, 2011; Di Serio et al., 2013; Wojciechowski y Cellary, 2013; Cózar et al., 2015), que expresan que los alumnos cuando interaccionan con objetos construidos en RA aumentan su motivación hacia la instrucción y muestran altos niveles de satisfacción en la interacción con estos objetos. Dicho de otra forma, los alumnos han mostrado 
una actitud positiva hacia estos recursos didácticos producidos en RA y ello puede favorecer el que puedan alcanzar resultados académicos con los mismos en su interacción (Wojciechowski y Cellary, 2013; Chung, Han y Joun, 2015).

Nuestro trabajo apunta también que estos materiales en los contextos de formación universitaria no son difíciles de manejar por los estudiantes, y ello creemos que es debido a la fuerte familiaridad que tienen con los dispositivos móviles, como ya hemos apuntado.

Por lo que se refiere al instrumento de análisis elaborado, señalar que el nivel de fiabilidad alcanzado nos sugiere que es de utilidad para recoger información de los alumnos respecto a la evaluación que realizan de objetos de aprendizaje tecnológico, y que los ítems que conforman las dos primeras dimensiones (aspectos técnicos y estéticos, y facilidad de utilización) pueden ser incorporados a otros instrumentos que se creen para una actividad similar.

Como todo estudio de investigación nuestro trabajo presenta también una serie de limitaciones que deben ser contempladas a la hora de interpretar correctamente nuestros hallazgos y la transferencia de los mismos, y los fundamentales desde nuestro punto de vista son: lo limitado del número de estudiantes y de objetos que se han puesto a disposición para su evaluación; que su evaluación aunque se ha llevado a cabo en los contextos de enseñanza posee cierta artificialidad, y que en el momento de la realización de la investigación la app de ios no estaba disponible, con lo cual algunos estudiantes tuvieron que seguir la experiencia con los dispositivos de los compañeros, lo que incorporaba un problema adicional por el conocimiento del dispositivo más los problemas que podría tener la observación conjunta. De todas formas, tiene el valor de comenzar una línea de investigación en RA la cual es muy deficitaria.

Por lo que se refiere a líneas futuras, nuestro trabajo, una vez analizadas con los estudiantes las valoraciones positivas que han realizado sobre los objetos, y que los perciben como fáciles de utilizar, van a ir en la línea de incorporarlos en situaciones reales de enseñanza-aprendizaje, donde se analicen las actitudes que los objetos desprenden en los estudiantes y su papel en la adquisición de rendimientos por parte de los estudiantes.

\section{REFERENCIAS BIBLIOGRÁFICAS}

Alaminos, A. (2006). El muestreo en la investigación social. En A. Alaminos y J. L. Castejón. Elaboración, análisis e interpretación de encuestas, cuestionarios y escalas de opinión (pp. 46-67). Alcoy: Marfil.

Avendaño, V.; Chao, C.; Mercado, M. ${ }^{a}$ M. y Bazán, O. (2012). La gestión del conocimiento en ambientes de aprendizaje que incorporan la realidad aumentada: el caso de la Universidad Virtual del Estado de Guanajuato en el nivel Bachillerato. Revista Educación y Futuro Digital, 2, 51-67.

Bongiovani, P. (2013). Realidad aumentada en la escuela: Tecnología, experiencias e ideas. Educ@contıc. Disponible en http://www.educacontic.es/blog/realidad. 
Bower, M. et al. (2014). Augmented Reality in education - cases, places and potentials. Educational Media International, 51 (1), 1-15.

Bressler, D. M. y Bodzin, A. M. (2013). A mixed methods assessment of students' flow experiences during a mobile augmented reality science game. Journal of Computer Assisted Learning, 29 (6), 505-517.

Cabero, J. (2016). Tendencias educativas para el siglo XXI. Madrid: Centro de Estudios Financieros.

Cabero, J. y Barroso, J. (2015). Realidad Aumentada: posibilidades educativas. En J. Ruiz-Palmero, J. Sánchez-Rodríguez y E. Sánchez-Rivas (Eds.). Innovaciones con tecnologías emergentes. Málaga: Universidad de Málaga.

Cabero, J. y Barroso, J. (2016). The educational possibilities of Augmented Reality. NAER. New Approaches in Educational Research, 5 (1), 44-50.

Cabero, J. y García, F. (Coords.) (2016). Realidad aumentada. Tecnología para la formación. Madrid: Síntesis.

Cabero, J. y Llorente, M. C. (2009). Actitudes, satisfacción, rendimiento académico y comunicación online en procesos de formación universitaria en blended learning. Revista Electrónica Teoría de la Educación: Educación y Cultura en la Sociedad de la Información, vol. 10, n. ${ }^{\circ}$. Descargado el día 6 de abril de 2009. http://www.usal.es/ teoriaeducacion/rev_numero_10_01/n10_01_cabero_llorente.pdf.

Cabero, J. y Marín, V. (2013). Valoración del entorno formativo universitario Dipro 2.0. Profesorado. Revista de Currículum y Formación del Profesorado, 17 (2), 369-383.

Carozza, L.; Tingdahi, D.; Bosché, F. y Gool, L. (2014). Markerless Vision-Based Augmented Reality for Urban Planning. Computer-Aided Civil and Infrastructure Engineering, 00, $1-16$.

Chang, H.; Wu, H. y Hsu, Y. (2013). Integrating a mobile augmented reality activity to contextualize student learning of a socioscientific issue. British Journal of Educational Technology, 44 (3), E95-E99. doi: 10.1111/j.1467-8535.2012.01379.x.

Chin-Ming, C. y Yen Nung, T. (2011). Interactive augmented reality system for enhancing library instruction in elementary schools. Computers and Education, 59, 638-652.

Chung, N.; Han, H. y Joun, Y. (2015). Tourists' intention to visit a destination: The role of augmented reality (AR) application for a heritage site. Computers in Human Behavior, 50, 588-599.

Coimbra, M.; Cardoso, T. y Mateus, A. (2015). Augmented Reality: an Enhancer for Higher Education Students in Math's learning? Procedia Computer Science, 67, 332-339. doi: 10.1016/j.procs.2015.09.277.

Cózar, R.; De Moya, M.; Hernández, J. y Hernández, J. (2015). Tecnologías emergentes para la enseñanza de las Ciencias Sociales. Una experiencia con el uso de Realidad Aumentada en la formación inicial de maestros. Digital Education Review, 27, 138-153.

De la Torre, J.; Martín-Dorta, N.; Saorín, J. L.; Carbonel, C. y Contero, M. (2013). Entorno de aprendizaje ubicuo con realidad aumentada y tabletas para estimular la comprensión del espacio tridimensional. RED, Revista de Educación a Distancia, 37. Descargado de http://www.um.es/ead/red/37.

De Pedro Carracedo, J. y Méndez, C. L. M. (2012). Realidad Aumentada: Una Alternativa Metodológica en la Educación Primaria Nicaragüense. IEEE-RITA, 7, 102-108.

Di Serio, A.; Ibáñez, M. B. y Delgado, C. (2013). Impact of an augmented reality system on students' motivation for a visual art course. Computers \& Education, 68, 586-596. doi:10.1016/j.compedu.2012.03.002. 
JULIO BARROSO OSUNA Y JULIO CABERO ALMENARA

EVALUACIÓN DE OBJETOS DE APRENDIZAJE EN REALIDAD AUMENTADA:

ESTUDIO PILOTO EN EL GRADO DE MEDICINA

Díaz, M. (2016). Augmented Reality Versus Virtual Reality: The Battle Is Real. Techcrunch. Descargado el día 8 de enero de 2016. http://techcrunch.com/2016/01/04/ar-vs-vr-thebattle-is-real/?ncid=rss.

Fombona, J.; Pascual M. ${ }^{a}$ A. y Madeira, M. ${ }^{a}$ F. (2012). Realidad aumentada, una evolución de las aplicaciones de los dispositivos móviles. Pixel-Bit. Revista de Medios y Educación, 41, 197-210.

Fracchia, C.; Alonso, A. y Martins, A. (2015). Realidad Aumentada aplicada a la enseñanza de Ciencias Naturales. Revista Iberoamericana de Educación en Tecnología y Tecnología en Educación, 16, 7-15.

Fundación Telefónica (2011). Realidad Aumentada: una nueva lente para ver el mundo. Madrid: Fundación Telefónica-Ariel.

Han, J.; Jo, M.; Hyun, E. y So, H. (2015). Examining young children's perception toward augmented reality-infused dramatic play. Education Technology Research Development, 63, 455-474. doi:10.1007/s11423-015-9374-9.

Johnson, L.; Adams Becker, S.; Cummins, M.; Estrada, V., Freeman, A. y Hall, C. (2016). NMC Horizon Report: 2016 Higher Education Edition. Austin, Texas: The New Media Consortium.

Kamarainen, A.; Metcalf, Sh.; Grotzer, T.; Browne, A.; Mazzuca, D.; Tutwiler, M. y Dede, Ch. (2013). EcomobiLE: Integrating augmented reality and probeware with environmental education field trips. Computers \& Education, 68, 545-556. doi:10.1016/j.compedu.2013.02.018

Ko, Ch.-H.; Chang, T.; Chen, Y. y Hua, L. (2011). The Application of Augmented Reality to Design Education. En M. Chang, W.-Y. Hwang, M.-P. Chen y W. Müller (Eds.). Edutainment Technologies. Educational Games and VirtualReality/Augmented Reality Applications (pp. 20-24). Heidelberg Berlin: Springer.

Liu, T. Y. (2009). A context-aware ubiquitous learning environment for language listening and speaking. Journal of Computer Assisted Learning, 25, 515-527.

Llorente, M. C. y Cabero, J. (2013). Blended learning: attitudes, satisfaction, academic performance and online communication in processes of University Training. The New Educational Review, 31 (1), 28-39.

Marín, V.; Cabero, J. y Barroso, J. (2014). Evaluando los entornos formativos online. El caso de DIPRO 2.0. REDU. Revista de Docencia Universitaria, 12 (2), 375-399.

Mateo, J. (2004). La investigación ex-post-facto. En R. Bisquerra (Coord.). Metodología de la investigación educativa (pp. 195-230). Madrid: La Muralla.

Mott, J.; Bucolo, S.; Cuttle, L.; Mill, J.; Hilder, M.; Miller, K. y Kimble, R. M. (2008). The efficacy of an augmented virtual reality system to alleviate pain in children undergoing burns dressing changes: A randomised controlled trial. Burns, 34, 803-808.

Muñoz, J. M. (2013). Realidad Aumentada, realidad disruptiva en las aulas. Boletín SCO$P E O$, 82. Disponible en http://scopeo.usal.es/realidad-aumentada-realidad-disruptiva-en-las-aulas/.

Neven A. M.; Hala, H. y Mohamed, I. (2011). ARSC: Augmented Reality Student Card An Augmented reality Solution for the educational field. Computers and Education, 56, 1045-1061. doi:10.1016/j.compedu.2010.10.019.

O'Dwyer, L. y Bernauer, J. (2014). Quantitative research for the qualitative researcher. California: Sage.

Pasaréti, O.; Hajdú, H.; Matuszka, T.; Jambori, A.; Molnar, I. y Turcsányi-Szabó, M. (2011). Augmented Reality in education. INFODIDACT 2011 Informatika Szakmódszertani Konferencia. Recuperado de http://people.inf.elte.hu/tomintt/infodidact_2011.pdf. 
JULIO BARROSO OSUNA Y JULIO CABERO ALMENARA

EVALUACIÓN DE OBJETOS DE APRENDIZAJE EN REALIDAD AUMENTADA ESTUDIO PILOTO EN EL GRADO DE MEDICINA

Prendes, C. (2015). Realidad aumentada y educación: análisis de experiencias prácticas. Pixel-Bit. Revista de Medios y Educación, 46, 187-203.

Quicios, M. P.; Ortega, I. y Trillo, M. P. (2015). Aprendizaje ubicuo de los nuevos aprendices y brecha digital formativa. Pixel-Bit. Revista de Medios y Educación, 46, 155-166.

Rodríguez, M. (2013). Experimentando la realidad aumentada. Integrando tecnología en el salón de clase. Disponible en http://mbintegrandotecnologia.blogspot.com.es.

Ruiz, D. (2011). La Realidad Aumentada y su dimensión en el arte: La obra aumentada. Arte y Políticas de Identidad, 5, 129-144.

Sabariego, M. (2012). El proceso de investigación (parte 2). En R. Bisquerra (Coord.). Metodología de la investigación educativa (pp. 127-163) (3. ${ }^{a}$ ed.). Madrid: La Muralla.

Santos, M.; Wolde, A.; Taketomi, T.; Yamamoto, G.; Rodrigo, M. ${ }^{a}$ M.; Sandor, C. y Kato, H. (2016). Augmented reality as multimedia: the case for situated vocabulary learning. Research and Practice in Techology Enhanced Learning, 11 (4), 1-23. doi:10.1186/ s41039-016-0028-2.

Sevillano, M. L. y Vázquez, E. (2015). Modelos de investigación en contextos ubicuos y móviles en Educación Superior. Madrid: McGraw-Hill-UNED.

Solano, C. A.; Casas, J. F. y Guevara, J. C. (2015). Aplicación móvil de realidad aumentada para la enseñanza de la clasificación de los seres vivos a niños de tercer grado. Ingeniería, 20 (1), 101-115.

Tecnológico de Monterrey (2015). Reporte EduTrends. Radar de Innovación Educativa 2015. Monterrey: Tecnológico de Monterrey.

Tsai, M.; Liu, P. y Yau, J. (2013). Using electronic maps and augmented reality based training materials as escape guidelines for nuclear accidents: An explorative case study in Taiwan. British Journal of Educational Technology, 44 (1), 18-21. doi:10.1111/j.14678535.2012.01325.x.

Vázquez, E. y Sevillano, M. L. (Eds.) (2015). Dispositivos digitales móviles en educación. El aprendizaje ubicuo. Madrid: Narcea.

Wojciechowski, R. y Cellary, W. (2013). Evaluation of learners' attitude toward learning in aries augmented reality environments. Computers \& Education, 68, 570-585. doi:10.1016/j. compedu.2013.02.014.

Wu, H.-S.; Wen-Yu, S.; Chang, H.-Y. y Liang, J. (2013). Current status, opportunities and challenges of augmented reality in education. Computers $\varepsilon$ Education, 62, 41-49. http:// dx.doi.org/10.1016/j.compedu.2012.10.024.

Yeo, C. T.; Ungi, T.; U-Thainual, P.; Lasso, A.; McGraw, R. C. y Fichtinger, G. (2011). The effect of augmented reality training on percutaneous needle placement in spinal facet joint injections. IEEE Transactions on Biomedical Engineering, 58 (7), 2031-2037. 